\title{
Flavobacterium meningosepticum infection: an epidemiological study in a newborn nursery
}

\author{
ML THONG, SD PUTHUCHEARY, AND EL LEE* \\ From the Department of Medical Microbiology, University Hospital, Kuala Lumpur, Malaysia
}

SUMMARY After an outbreak of flavobacterium meningitis in the newborn nursery of the University Hospital, an investigation was carried out to determine the possible sources of this organism in the nursery. Various serotypes of the organism were recovered from a variety of sources such as wash basins, sinks, suction apparatus, and disinfectants in the nursery as well as the neighbouring wards. Colonisation of the pharynx with this organism was demonstrated in several clinically healthy babies in the nursery. The possible role of fomites, hospital staff, newborn babies, and their mothers in the transmission of the organism is considered together with the measures taken to reduce environmental contamination.

Flavobacterium meningosepticum, a Gram-negative water bacillus, is sometimes responsible for epidemic meningitis in newborn infants in hospital nurseries. ${ }^{1-6}$ The infection in babies tends to be severe with a high mortality rate and serious neurological sequelae in the surviving patients. Brody et al. ${ }^{1}$ described two outbreaks of meningitis caused by $F$. meningosepticum affecting primarily premature infants in a hospital nursery. This outbreak was described as hospitalacquired, but attempts to isolate the organism from human contacts were unsuccessful. Cabrera and Galen $^{2}$ reported an outbreak of 14 cases of neonatal meningitis due to $F$. meningosepticum. The source was traced to a leaking sink trap in an anteroom of the premature nursery. Repair of the faulty leaky trap eradicated the reservoir of this infection and terminated the outbreak. George et al. ${ }^{3}$ also reported 14 cases of meningitis and 30 asymptomatic nasopharyngeal carriers during an outbreak in their newborn nursery. Plotkin and McKitrick ${ }^{5}$ described two cases of flavobacterium meningitis which were traced to a saline solution. This saline solution containing flavobacteria was stored in a glass bottle that was seldom sterilised but refilled with sterile saline as needed. The solution was aspirated into a rubber bulb and used to flush the eyes of infants after the application of silver nitrate solution. More recently, Coyle-Gilchrist et al. ${ }^{7}$ isolated $F$. meningosepticum

* Present address: Assunta Hospital, Petaling Jaya, Malaysia. Accepted for publication 3 September 1980 from aqueous chlorhexidine gluconate (Hibitane) solutions used in the wards for the storage of thermometers and for routine disinfection.

King, ${ }^{8}$ at the Center for Disease Control, Atlanta, USA, used serological procedures for typing strains isolated in epidemiological studies. Six serotypes (A to $F$ ) have been described, type $C$ being responsible for most of the cases of meningitis. 489

\section{Clinical cases}

Table 1 presents the relevant data on seven confirmed cases of flavobacterium meningitis acquired in the University Hospital since 1972. F. meningosepticum was isolated from the cerebrospinal fluid in all seven infants, from blood in three infants, and from peritoneal fluid in one infant (case 6). Four strains were found to be serotype $C$, two were of serotype $F$ (cross-reacting with serotype E), while one strain could not be classified into any of the six serotypes.

Cases 1 and 2 who died did not receive intraventricular chemotherapy. The other babies received appropriate antibiotics such as rifamycin SV, erythromycin, or novobiocin, for it has been shown that these organisms have an unusual pattern of antibiotic sensitivity, being generally resistant to the conventional chemotherapeutic agents used in the treatment of neonatal meningitis, that is, ampicillin, gentamicin, kanamycin, and chloramphenicol.1011 The treated babies who survived the infection are being followed up. Cases 3, 4, and 5 are normal 
Table 1 Data on neonates with flavobacterium meningitis acquired in the University Hospital, Kuala Lumpur

\begin{tabular}{|c|c|c|c|c|c|c|}
\hline Case & $\begin{array}{l}\text { Time of } \\
\text { illness }\end{array}$ & $\begin{array}{l}\text { Birth weight } \\
(g)\end{array}$ & $\begin{array}{l}\text { Age at } \\
\text { onset } \\
\text { (days) }\end{array}$ & Clinical presentation & Serotype & Outcome \\
\hline $\begin{array}{l}1 * \\
2 * \\
3 \\
4 \\
5 \\
6 \\
7\end{array}$ & $\begin{array}{l}\text { Feb '72 } \\
\text { July'74 } \\
\text { May'75 } \\
\text { June'76 } \\
\text { Aug'76 } \\
\text { Sept'76 } \\
\text { April'77 }\end{array}$ & $\begin{array}{l}2060 \\
2500 \\
3300 \\
2750 \\
2430 \\
1840 \\
1980\end{array}$ & $\begin{array}{l}6 \\
4 \\
3 \\
6 \\
6 \\
3\end{array}$ & $\begin{array}{l}\text { Fever, tachypnoea, circulatory collapse } \\
\text { Fever, lethargy, tachypnoea } \\
\text { Fever, convulsions, bulging fontanelle } \\
\text { Fever, convulsions } \\
\text { Fever, irritability, jitteriness } \\
\text { Peritonitis, fever, convulsions } \\
\text { Fever, jitteriness }\end{array}$ & $\begin{array}{l}\text { C } \\
\text { C } \\
\text { C } \\
\text { F(E) } \\
\text { F(E) } \\
\text { Unclassified } \\
\text { C }\end{array}$ & $\begin{array}{l}\text { Died } \\
\text { Died } \\
\text { Recovered } \\
\text { Recovered } \\
\text { Recovered } \\
\text { Hydrocephalus, mental retardation, spasticity } \\
\text { Hydrocephalus }\end{array}$ \\
\hline
\end{tabular}

*Intraventricular antibiotics were not administered in these patients.

( ) Serogroup cross-reaction.

neurologically. Case 6 has hydrocephalus with severe brain damage; the infection in this infant began with umbilical sepsis and peritonitis. The infecting organism was isolated from the peritoneal fluid two days before the cerebrospinal fluid yielded the same organism. Case 7 also has hydrocephalus without showing any other evidence of neurological handicap. This infant, the elder of twin boys, was nursed in a cot alongside his twin who was in an incubator in the nursery. The younger twin did not become ill, and $F$. meningosepticum was not isolated from his throat.

The first four cases were widely separated in time and were not thought to be related. However, case 5 occurred within three months of case 4 , and the infecting organisms were found to be identical biochemically as well as serologically. It was also noted that case 5 occupied an incubator used earlier by case 4. Accordingly, an investigation was undertaken to discover the possible source of the infections. When case 6 and, later, case 7 occurred due to other serotypes, the epidemiology became more complex than that involving a single serotype.

\section{Epidemiological investigations}

\section{BACKGROUND}

Approximately 400 live babies delivered in this hospital each month are accommodated in three areas of the maternity block. Infants who appear normal at birth are nursed next to their mothers in one of two postnatal wards. Those from premature or complicated and septic deliveries (about 60 a month) are admitted to the special care nursery which can accommodate a maximum of 19 babies. The special care nursery has two big rooms, nursery I and nursery II, to accommodate eight babies each, and three single isolation rooms for infected babies.

\section{BACTERIOLOGY}

In order to detect $\boldsymbol{F}$. meningosepticum in the environment, equipment, and various fomites and throat swabs of staff and patients, a large number of samples were collected for examination from the $\stackrel{\omega}{+}$ nursery and neighbouring wards. These materials were plated on to ox or human blood agar (blood agar base (Oxoid) with $10 \%$ blood), McConkey agar 0 (Oxoid), and modified Thayer Martin medium (BBL) containing vancomycin $3 \mu \mathrm{g} / \mathrm{ml}$, colistin $7.5 \mu \mathrm{g} / \mathrm{ml}$, $\rightarrow$ nystatin 1250 units $/ \mathrm{ml}$, and trimethroprim lactate $5 \mu \mathrm{g} / \mathrm{ml}$.

Surface areas in the nursery, such as incubators, $\overrightarrow{\mathscr{O}}$ which do not contain any water for humidification, $\stackrel{-}{-}$ bassinets, weighing machines, suction tubings, and sinks, were sampled using swabs premoistened with $\sum$ sterile nutrient broth. After collection, the swabs were $\bar{\partial}$ inoculated on to agar plates and/or into nutrient broth (Oxoid). Aliquots of fluids from various $\stackrel{\AA}{\propto}$ sources were cultured directly in nutrient broth. $\overrightarrow{\vec{O}}$ After one to two days' incubation, the broth cultures were plated on to blood agar and McConkey agar. Disinfectants were examined using the in-use test of Kelsey and Maurer. ${ }^{12}$ Culture plates were exposed for air sampling for $\mathbf{4}$ hours at different times of the day in various locations in the nursery. The ward air was also cultured using a slit sampler on several occasions. More than 1450 culture specimens were examined between August 1976 and June 1977, in- $₹$ cluding 91 throat swabs from 46 newborn babies in 을 the nursery. In addition, 585 sputum specimens and $D$ 475 vaginal swabs sent for routine culture between February and August 1977 were also plated on $N$ modified Thayer Martin medium to detect $F$. meningosepticum as a supplement to our search for $N$ the organisms biologically identical with the strains $\mathrm{\omega}$ causing meningitis in our newborn babies. ${ }^{13}$

Tap water from several wards was collected for 0 filtration through sterile disposable membrane filters of $0.45 \mu$ pore size (Falcon). After filtration the mem- $\stackrel{?}{?}$ branes were transferred to agar plates. The agar $T$ plates and broth cultures were incubated aerobically at $37^{\circ} \mathrm{C}$ for two to seven days. Suspicious-looking $\cong$ colonies were picked out and inoculated into tubes $\stackrel{\square}{\triangle}$ of Kliger iron agar (Oxoid). Organisms not ferment- 
ing glucose were subcultured on blood agar and identified specifically using the methods of Cowan and Steel. ${ }^{14}$

The strains were serotyped by the rapid slide agglutination test using absorbed rabbit antiFlavobacterium meningosepticum sera (Difco). The polyvalent antiserum was used first, and if the strain was found to be positive then the individual group (A to F) sera were used.

\section{Results}

$F$. meningosepticum was recovered from various sources in the nursery as well as in the neighbouring paediatric and obstetric wards. These sources are shown in Table 2. The organism was recovered mainly from the outlets of hand basins, sinks, and babies' bath basins as well as from stock bottles of aqueous chlorhexidine. Freshly distilled water gave negative results but stored distilled water in the pharmacy unit yielded one strain serotype $E$ and one unclassified strain. The organism was also detected in the throat cultures of four neonates and of two postnatal mothers. In two of the babies the organism was recovered on several occasions. All four babies and the two mothers were clinically healthy.

$F$. meningosepticum was recovered from 67 (11\%) of 585 routine sputum specimens cultured on modified Thayer Martin medium. These specimens were collected from adult outpatients and inpatients of the hospital. Among the $\mathbf{4 7 5}$ vaginal swabs cultured, only one strain of $F$. meningosepticum was recovered, that is, an isolation rate of $0.2 \%$. This strain, which could not be serogrouped, was recovered from a 28year-old outpatient with puerperal fever.

A number of other sources examined in the special care nursery failed to yield the organism. These were

Table 2 Positive isolation of Flavobacterium meningosepticum from various sources

\begin{tabular}{lccc}
\hline Source & $\begin{array}{l}\text { Number } \\
\text { examined }\end{array}$ & $\begin{array}{c}\text { Number } \\
\text { positive }\end{array}$ & $\begin{array}{c}\text { Percent } \\
\text { positive }\end{array}$ \\
\hline $\begin{array}{l}\text { Suction apparatus of babies' } \\
\text { resuscitation machines }\end{array}$ & 2 & 2 & - \\
$\begin{array}{l}\text { Babies' bath basins } \\
\text { Hand basins in paediatric and }\end{array}$ & 204 & 73 & 100 \\
$\quad \begin{array}{l}\text { obstetric wards } \\
\text { Aqueous solutions of chlorhexidine }\end{array}$ & 19 & 12 & 36 \\
$\begin{array}{l}\text { (0.05\%, 0.5\% 1\%) } \\
\text { Stored distilled water in pharmacy }\end{array}$ & 1 & 1 & 63 \\
$\begin{array}{l}\text { Bpecial care nursery ward air } \\
\text { Thronchial suction tubings* }\end{array}$ & 8 & 1 & $-12 \cdot 5$ \\
Throat swabs of newborn babies & 46 & 4 & 10 \\
Routine sputum specimens & 49 & 4 & $8 \cdot 7$ \\
Routine high vaginal swabs & 585 & 67 & $4 \cdot 1$ \\
\hline
\end{tabular}

*After use in special care nursery and labour ward. rectal thermometers, brushes and mops, refrigerators, and air vents of air-conditioners. $F$. meningosepticum was not isolated from any of the incubators used by newborn babies during the 10-month period of study; 512 samples were tested. None of the incubators used in this hospital is humidified, that is, there is no water reservoir inside each incubator. Repeated throat cultures of 24 nursery staff also failed to yield the organism.

Table 3 shows the serotypes of the isolated strains by source. All the serotypes associated with the seven cases of neonatal meningitis have been recovered from the various sources examined in the study. This includes the $F$. meningosepticum recovered from case 6 and labelled as unclassified (that is, not group A, B, C, D, E, F, or G) which was also isolated from hand basins in the paediatric and obstetric wards, bronchial suction tubings, and routine sputum specimens and routine vaginal swabs. Thus widespread possible sources of infection were found in the nursery and neighbouring wards.

\section{Discussion}

Over a period of six years (June 1971 to June 1977), 16 cases of neonatal meningitis due to $F$. meningosepticum were seen at the University Hospital, Kuala Lumpur. Of these 16 cases, only seven were acquired in the University Hospital; the rest were from eight different hospital environments throughout West Malaysia.

$F$. meningosepticum has high virulence and selectivity for the newborn, with a predilection for premature infants. However, not all neonates exposed to the organism became ill. Cabrera and Galen ${ }^{2}$ reported that only one twin of each of three sets became ill and died, while the other twins neither became ill

Table 3 Serotypes of Flavobacterium meningosepticum by source

\begin{tabular}{|c|c|c|c|c|c|c|c|}
\hline \multirow[t]{2}{*}{ Source } & \multicolumn{7}{|c|}{ Serotype } \\
\hline & $A$ & $\boldsymbol{B}$ & $C$ & $\boldsymbol{D}$ & $\boldsymbol{E}$ & $\boldsymbol{F}$ & Unclassified \\
\hline CSF & - & - & 4 & - & (2) & 2 & 1 \\
\hline Babies' resuscitation machines & - & - & - & - & 2 & (1) & - \\
\hline Babies' bath basins & - & - & - & - & 4 & - & - \\
\hline $\begin{array}{l}\text { Hand basins in paediatric and } \\
\text { obstetric wards }\end{array}$ & 4 & 27 & 4 & 3 & 16 & 2 & 17 \\
\hline Aqueous solutions of chlorhexidine & - & 2 & 1 & 4 & 4 & 1 & - \\
\hline Stored distilled water in pharmacy & - & - & - & - & 1 & - & 1 \\
\hline Special care nursery ward air & 1 & - & - & - & - & - & - \\
\hline Bronchial suction tubings & - & - & 1 & - & 2 & - & 1 \\
\hline Throat swabs of newborn babies & - & - & 2 & - & 2 & - & - \\
\hline Throat swabs of postnatal mothers & - & - & - & - & 2 & - & - \\
\hline Routine sputum specimens & 3 & 2 & 7 & - & 47 & - & 8 \\
\hline Routine vaginal swabs & - & - & - & - & - & - & 1 \\
\hline
\end{tabular}


nor were colonised by this organism. This suggests that immune mechanisms may play a role in these nosocomial infections. That compromised adults can also become ill and die as a result of infection with this opportunist organism has been reported by Mani et al. ${ }^{15}$

Isolates from four of our cases (Table 1) were found to belong to serotype $\mathrm{C}$, the commonest serotype causing infections in the newborn.489 One isolate (case 6 ) was identified as $F$. meningosepticum on the basis of its morphology and biochemical properties but could not be classified as it did not react with any of the $F$. meningosepticum antisera. Isolates from cases 4 and 5 were found to belong to serotype $F$ but showed cross-reaction with serotype E. These serological reactions were confirmed by Dr RE Weaver, Center for Disease Control, Atlanta, USA.

Our relatively high isolation rates of $F$. meningosepticum from environmental sources were due to our use of modified Thayer Martin medium as a selective medium for this organism. These strains of $F$. meningosepticum were mainly present in samples taken from babies' bath basins, handbasins in the paediatric and obstetric wards (73 positives out of 204 samples), and aqueous solutions of chlorhexidine (12 positives out of 19 samples). All six serotypes as well as serologically untypable strains were isolated from various sources, including one from a babies' bath basin belonging to serotype $\mathrm{E}$ but cross-reacting with serotype F. Type E was the commonest serotype isolated. These results strongly suggest that flavobacterium infections are acquired not from mothers or nursery personnel but from environmental contamination and accounted for the few and sporadic cases seen in our hospital. It is known that outbreaks caused by environmental contamination tend to be intermittent and affect only a few babies in a nursery. ${ }^{13}$ Our investigations also show that flavobacterium infections can become endemic in a particular hospital. Only two of our cases were proven to be related epidemiologically (cases 4 and 5).

We are unable to reach conclusions regarding the portals of entry in our patients, although in one patient (case 6) the organism probably gained entry via the infected umbilicus. Direct invasion of the organism through the mucous membranes of the respiratory tract, conjunctiva, or abraded skin are possible portals of entry. 2516

It must be emphasised that vigorous control measures must be instituted to contain and prevent further infections. Bacteriological surveillance of equipment and fomites, disinfectants, distilled water, and anything that comes in contact with the newborn must be carried out regularly. The human factor must also be considered, and periodic supervision $\stackrel{0}{\overrightarrow{7}}$ and observation of manoeuvres and aseptic tech- $\underline{\underline{0}}$ niques carried out by the doctors and nursing staff is also important.

\section{CONTROL MEASURES}

Control of infection in the newborn intensive care $\stackrel{\mathbb{D}}{\Omega}$ unit requires an understanding of the reservoirs and modes of transmission of nosocomial organisms. As $\vec{\circ}$ a result of the investigation carried out by us, several changes in the existing facilities and procedures were $\vec{\omega}$ made in the nursery and obstetric wards. The suction $\stackrel{\circ}{\circ}$ apparatus of resuscitation machines was dismantled, cleaned thoroughly with soap and water, and disin- $\omega$ fected with $1 \%$ chlorhexidine in $70 \%$ spirit after each day of use. With this method of disinfection rubber tubings became brittle and had to be replaced at $\mathcal{O}$ frequent intervals. Suction bottles were changed $\frac{\text { ? }}{2}$ daily.

The current procedures for cleaning with soapy $D$ water and disinfection with Amphyl solutions for incubators and bronchial suction apparatus were $\vec{\varphi}$ found to be satisfactory. Amphyl solution, produced $\stackrel{\infty}{\longrightarrow}$ by Sterling Drugs, USA, is described by the manufacturer as a phenolic disinfectant, deodorant, and detergent. The concentrations of Amphyl solutions used were $0.5 \%$ for incubators and $2 \%$ for bronchial suction apparatus, as recommended by the manufacturer.

Nasal suction catheters used for 24 hours were $\overrightarrow{\vec{O}}$ soaked in aqueous chlorhexidine. Aqueous solutions 3 of chlorhexidine were not used as disinfectants for the skin or for any equipment unless they were supplied as heat-sterilised solutions in small-volume containers. Bath basins, wash basins, and sinks were cleaned with scouring powder at least twice daily. Since flavobacteria are often found in water, ${ }^{2}$ only boiled water that has been cooled in sterile containers was used to wash premature babies in the nursery.

Handwashing ${ }^{17}$ can reduce the transmission of nosocomial disease, and it was recommended that $\frac{7}{0}$ sterile aqueous chlorhexidine be used for handwashing during doctors' ward rounds in between $N$ examination of babies, especially high-risk neonates. $N$ Washing of hands with soap and running water was sufficient elsewhere in the wards. The use of paper ${ }^{\mathrm{\omega}}$ towels or hot air for the drying of hands was also recommended.

Samples for bacteriological examination were taken periodically to determine the degree of con-tamination in the environment and equipment.

Since April 1977, when case 7 occurred, no other? case of neonatal meningitis due to $F$. meningosepti- $\frac{\bigcirc}{\mathbb{D}}$ cum has occurred in this hospital since the controlo measures were taken to prevent further infections. 
We are grateful for the assistance of Staff Nurse Patricia Tan, of the Hospital Infection Team, and for the cooperation of the nursing staff of the paediatric and obstetric units. We are also indebted to Dr RE Weaver, of CDC, Atlanta, USA, for examining our organisms, and to Dr MT Parker, Central Public Health Laboratory, Colindale, London, for helpful criticisms during the preparation of this paper.

This study was supported by a University of Malaya Vote F Grant, Number 73/77.

Part of this paper was presented at the 12th Singapore-Malaysia Congress of Medicine held in Singapore on 28-31 July 1977.

\section{References}

${ }^{1}$ Brody JA, Moore H, King EO. Meningitis caused by an unclassified gram-negative bacterium in newborn infants. Am J Dis Child 1958;96:1-5.

2 Cabrera HA, Galen HD. Epidemic meningitis of the newborn caused by flavobacteria. I. Epidemiology and bacteriology. Am J Dis Child $1961 ; 101: 289-95$.

${ }^{3}$ George RM, Cochran CP, Wheeler WE. Epidemic meningitis of the newborn caused by flavobacteria. II. Clinical manifestations and treatment. Am J Dis Child $1961 ; 101$ : 296-304.

${ }^{4}$ Seligmann R, Komarov M, Reitler R. Flavobacterium meningosepticum in Israel. Br Med J 1963 ;ii:1528-9.

5 Plotkin SA, McKitrick JC. Nosocomial meningitis of the newborn caused by a flavobacterium. JAMA 1966;198: 662-4.

- Madruga M, Zanon U, Pereira CMN, Galvao AC. Meningitis caused by Flavobacterium meningosepticum. The first epidemic outbreak of meningitis in the newborn in South America. J Infect Dis 1970;121:328-30.

${ }^{7}$ Coyle-Gilchrist MM, Crewe P, Roberts G. Flavobacterium meningosepticum in the hospital environment. J Clin Pathol 1976;29:824-6.

${ }^{8}$ King EO. Studies on a group of previously unclassified bacteria associated with meningitis in infants. Am J Clin Pathol 1959;31:241-7.

9 Agarwal KC, Ray M. Meningitis in a newborn due to Flavobacterium meningosepticum. Indian J Med Res 1971; 59:1006-9.

${ }^{10}$ Lee EL, Robinson MJ, Thong ML, Puthucheary SD. Rifamycin in neonatal flavobacteria meningitis. Arch Dis Child 1976;51:209-13.

11 Lee EL, Robinson MJ, Thong ML, Puthucheary SD, Ong $\mathrm{TH}, \mathrm{Ng} \mathrm{KK}$. Intraventricular chemotherapy in neonatal meningitis. J Pediatr 1977;91:991-5.

${ }^{12}$ Kelsey JC, Maurer IM. An in-use test for hospital disinfectants. Monthly Bull Minist Health Lab Service 1966; 25:180-4.

13 Anonymous. Water bugs in the bassinet. Am J Dis Child $1961 ; 101: 273-7$.

14 Cowan ST, Steel KJ. Manual for the identification of medical bacteria. 2nd ed. Cambridge: Cambridge University Press, 1974.

15 Mani RM, Kuruvila KC, Batiwala PM, Damle PN, Shirgaonkar GV, Soni RP, Vyas PR. Flavobacterium meningosepticum as an opportunist. J Clin Pathol 1978; 31:220-2.

${ }^{16}$ Sugathadasa AA, Arseculeratne SN. Neonatal meningitis caused by new serotype of Flavobacterium meningosepticum. Br Med J $1963 ; \mathbf{i}: 37-8$.

17 Goldmann DA. Nosocomial infection-a hazard of newborn intensive care. $N$ Engl J Med 1976;294:1342-3.

Requests for reprints to: Dr SD Puthucheary, Department of Medical Microbiology, University Hospital, Kuala Lumpur, Malaysia. 